White Dwarfs as Probes of Fundamental Physics and Tracers of

Planetary, Stellar, and Galactic Evolution

Proceedings IAU Symposium No. 357, 2019

(c) 2019 International Astronomical Union

***NAME OF EDITORS*** DOI: $00.0000 / \mathrm{X} 000000000000000 \mathrm{X}$

\title{
The Search for Planet and Planetesimal Transits of White Dwarfs with the Zwicky Transient Facility
}

\author{
Keaton J. Bell ${ }^{1}$ \\ ${ }^{1}$ NSF Astronomy and Astrophysics Postdoctoral Fellow and DiRAC Institute Fellow, \\ Department of Astronomy, University of Washington, Seattle, WA 98195, USA \\ email: keatonb@uw.edu
}

\begin{abstract}
Planetary materials orbiting white dwarf stars reveal the ultimate fate of the planets of the Solar System and all known transiting exoplanets. Observed metal pollution and infrared excesses from debris disks support that planetary systems or their remnants are common around white dwarf stars; however, these planets are difficult to detect since a very high orbital inclination angle is required for a small white dwarf to be transited, and these transits have very short (minute) durations. The low odds of catching individual transits could be overcome by a sufficiently wide and fast photometric survey. I demonstrate that, by obtaining over 100 million images of white dwarf stars with 30-second exposures in its first three years, the Zwicky Transient Facility (ZTF) is likely to record the first exoplanetary transits of white dwarfs, as well as new systems of transiting, disintegrating planetesimals. In these proceedings, I describe my project strategy to discover these systems using the ZTF data.
\end{abstract}

Keywords. planetary systems, white dwarfs, surveys

\section{Introduction}

White dwarf stars (WDs) represent the final evolutionary stage of $>97 \%$ of stars (e.g., Williams et al. 2009), including the host stars of every known transiting exoplanet. The present population of planets orbiting WDs reveals the fates of these planets that missions like Kepler have shown to be ubiquitous around main sequence stars (e.g., Hsu et al. 2019). By virtue of its high volumetric survey speed (Bellm 2016), the Zwicky Transient Facility (ZTF) holds the promise of detecting the first members of this important exoplanet population, the details of which will inform our theories of planetary migration and survival in post-main-sequence circumstellar environments.

Despite the fact that no intact planets orbiting WDs have been previously identified, we have compelling observational evidence that such planets are common. The high gravities of WDs cause metals to diffuse quickly below their photospheres (Koester \& Wilken 2006), yet 27-50\% of WDs are metal polluted (Koester et al. 2014), implying that accretion of metals is ongoing. Chemical abundance analyses support that these metals come from rocky planetary debris (e.g., Gänsicke et al. 2012). Furthermore, debris disks are detected via infrared excesses from $\approx 2 \%$ of these systems (Rebassa-Mansergas et al. 2019). Recently, disintegrating planetesimals have even been discovered to orbit a couple of specific metal-polluted WDs (Vanderburg et al.|2015; Manser et al. 2019). Some of my own high speed photometry from McDonald Observatory of the transiting debris system WD $1145+017$ is displayed in Figure 1.

Detecting planets around WDs is observationally very challenging since transits of these roughly Earth-sized stars have short ( minute) durations and require nearly edgeon alignments. Still, transits of WDs can be very deep (even occulting), producing unmis- 


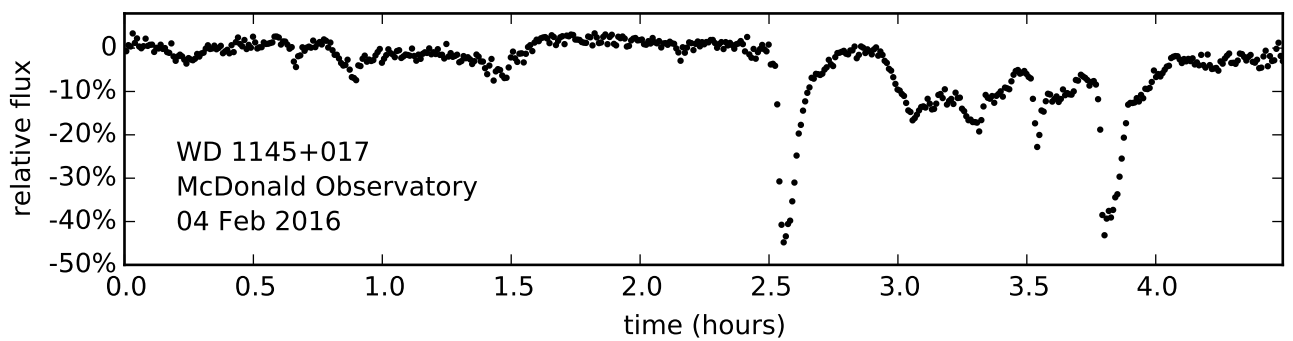

Figure 1. WD 1145+017 light curve from McDonald Observatory covering one orbital period.

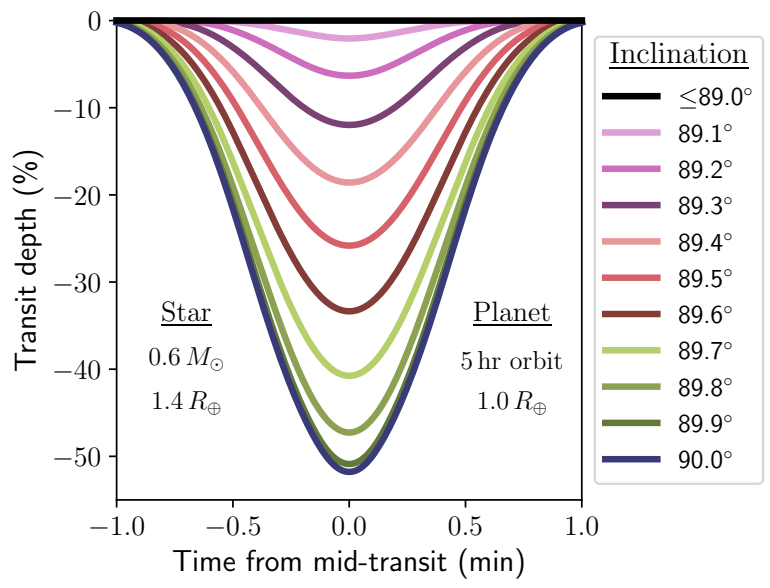

Figure 2. Model light curves of a $1 R_{\oplus}$ planet transiting a WD at different inclination angles, simulated with the BATMAN package (Kreidberg 2015) accounting for 30-second ZTF exposures.

takable signals in well-timed images. Figure 2 explores the transit profile of an Earth-sized planet in a 5-hour orbit around a typical $0.6 \mathrm{M}_{\odot} \mathrm{WD}$ at very high inclination angles.

Previous searches for such dips in samples of $\sim 1000$ WDs have yielded no transit detections (from, e.g., WASP, Faedi et al. 2011; Pan-STARRS, Fulton et al. 2014; K2, van Sluijs \& Van Eylen 2018; DECam, Dame et al. 2019, GALEX, Rowan et al.|2019). Discovering the first planets to transit WDs will require orders of magnitude larger sample sizes. Cortés, \& Kipping (2019) and Lund et al. (2018) have simulated the expected yield for WD transits after 10 years of Large Synoptic Survey Telescope (LSST) operations (ca. 2033), anticipating 1000 detections. Because LSST is a deep survey, opportunities to follow-up candidate WD transits with large telescopes will be extremely limited. The magnitude range of $\mathrm{ZTF}$, on the other hand, is readily accessible to more modest follow-up facilities, and I demonstrate in these proceedings that the current potential for discovering WD transits with the modern ZTF survey has been largely overlooked.

\section{Detectability of Planet Transits with ZTF}

Thanks to Gaia astrometry, we now know the identities (Gentile Fusillo et al. 2019) of most of the $\approx 220,000$ WDs in the ZTF survey footprint (declination $>-31 \mathrm{deg}$ ) brighter than the median $5 \sigma$ survey depth of $g=20.8 \mathrm{mag}$, each of which will receive a median $\approx 870$ observations over three years (Bellm 2014). I use the BATMAN package (Kreidberg 


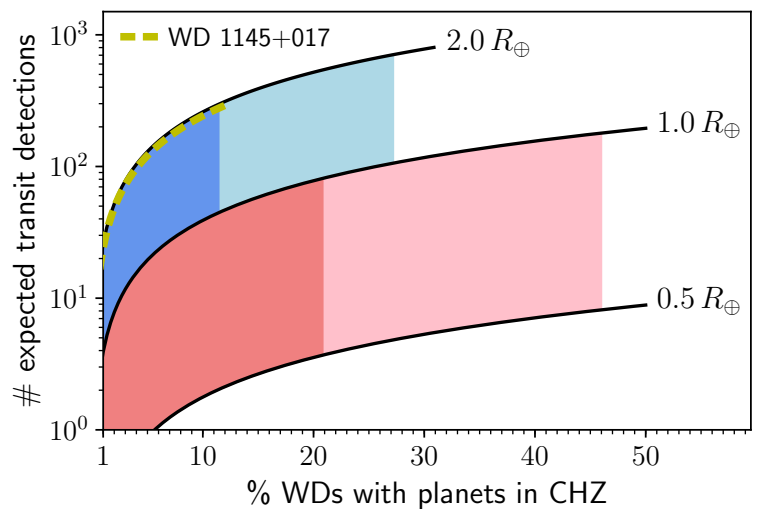

Figure 3. Simulated number of transit detections expected from the three-year ZTF survey for different percentages of WDs hosting $0.5,1$, and $2 R_{\oplus}$ planets in the continuously habitable zone. Regions are shaded below the $95 \%$ (light) and $68 \%$ (dark) confidence upper limits on planet occurrence from van Sluijs \& Van Eylen (2018). The dashed line shows the expected detections of disintegrating planetesimals transiting WDs based on the first such system, WD 1145+017.

2015) to synthesize transit light curves for planets that uniformly and isotropically populate the period range of the continuously habitable zones $\left(4.5 \mathrm{hr}<P_{\text {orb }}<32 \mathrm{hr}\right.$; Agol 2011) around typical $0.6 \mathrm{M}_{\odot}$ WDs (e.g., Tremblay et al. 2013). A transit detection is considered significant when the measured flux decrease exceeds a $5 \sigma$ threshold, where $\sigma$ is the photometric error achieved through the magnitude range observed by ZTF (Bellm et al. 2019).

Figure 3 displays the expected WD planet yield from ZTF. The solid lines show the number of $5 \sigma$ transit detections for a given fraction of WDs that host planets of radii $0.5,1$, and $2 R_{\oplus}$ in their continuously habitable zones. The shaded regions are consistent with recent constraints based on $\sim 1000$ non-detections from K2 van Sluijs \& Van Eylen 2018). If as few as $1 \%$ of WDs host $1-2 R_{\oplus}$ planets in their continuously habitable zones, $\mathrm{ZTF}$ will detect $\gtrsim 10$ exoplanet transits during its 3-year survey.

$\mathrm{ZTF}$ is also expected to discover many new examples of disintegrating planetesimal systems transiting WDs like WD $1145+017$, which was found in K2 data (Vanderburg et al. 2015). Using the ground-based light curve of this system obtained with 30-second exposures from the McDonald Observatory 2.1-meter Otto Struve Telescope (Figure 1) as a template, I include estimates of the number of similar systems that ZTF will detect with the dashed line in Figure 3 . In fact, the second such transiting planetesimal system has already been discovered from the first public release of ZTF data (ZTF J013906.17+524536.89; Vanderbosch et al. 2019).

\section{Project Strategy}

This work is the topic of the project "Discovering the First Exoplanets Around White Dwarf Stars with the Zwicky Transient Facility" that was granted a three-year NSF Astronomy and Astrophysics Postdoctoral Fellowship award \#1903828 (PI: Bell). The project is designed with the following strategy:

(a) Use the Astronomy eXtensions for Spark (AXS; Zečević et al. 2019) tools to efficiently identify candidate transits of likely WDs in the large ZTF data set (including both public and proprietary partnership data). 
(b) After validating the data quality of ZTF measurements that are consistent with significant brightness decreases, obtain follow-up high-speed photometry from Apache Point Observatory (APO), confirming the transits and resolving their shapes and periods.

(c) Obtain spectra of the WD hosts at APO to characterize stellar atmospheric parameters $\left(T_{\text {eff }}, \log g\right)$ and to rule out binarity for targets that exhibit light curves consistent with binary eclipses.

(d) Begin long-term monitoring of short-period transits to measure potential timing variations that probe the population of more distant planets that are unlikely to transit.

(e) Also identify signatures of transits of WDs by extended planetesimal debris (such as WD 1145+017, Vanderburg et al. 2015; ZTF J013906.17+524536.89, Vanderbosch et al. 2019), and begin long-term photometric monitoring for dynamical variations (e.g., Gänsicke et al. 2016).

$(f)$ At the conclusion of the three-year ZTF survey, use the total number of transit detections and non-detections to place strict constraints on the occurrence rates of planets of different sizes and orbital periods around WDs.

\section{Acknowledgements}

This material is based upon work supported by the National Science Foundation under Award No. 1903828.

\section{References}

Agol, E. 2011, ApJL, 731, L31

Bellm, E. 2014, The Third Hot-wiring the Transient Universe Workshop, 27

Bellm, E. C. 2016, PASP, 128, 084501

Bellm, E. C., Kulkarni, S. R., Graham, M. J., et al. 2019, PASP, 131, 018002

Cortés, J., \& Kipping, D. 2019, MNRAS, 488, 1695

Dame, K., Belardi, C., Kilic, M., et al. 2019, MNRAS, 490, 1066

Faedi, F., West, R. G., Burleigh, M. R., Goad, M. R., \& Hebb, L. 2011, MNRAS, 410, 899

Fulton, B. J., Tonry, J. L., Flewelling, H., et al. 2014, ApJ, 796, 114

Gänsicke, B. T., Aungwerojwit, A., Marsh, T. R., et al. 2016, ApJL, 818, L7

Gänsicke, B. T., Koester, D., Farihi, J., et al. 2012, MNRAS, 424, 333

Gentile Fusillo, N. P., Tremblay, P.-E., Gänsicke, B. T., et al. 2019, Monthly Notices of the Royal Astronomical Society, 482, 4570

Hsu, D. C., Ford, E. B., Ragozzine, D., et al. 2019, AJ, 158, 109

Koester, D., Gänsicke, B. T., \& Farihi, J. 2014, A\&A, 566, A34

Koester, D., \& Wilken, D. 2006, A\&A, 453, 1051

Kreidberg, L. 2015, PASP, 127, 1161

Lund, M. B., Pepper, J. A., Shporer, A., et al. 2018, arXiv e-prints, arXiv:1809.10900

Manser, C. J., Gänsicke, B. T., Eggl, S., et al. 2019, Science, 364, 66

Rebassa-Mansergas, A., Solano, E., Xu, S., et al. 2019, MNRAS, 489, 3990

Rowan, D. M., Tucker, M. A., Shappee, B. J., et al. 2019, MNRAS, 486, 4574

Tremblay, P.-E., Ludwig, H.-G., Steffen, M., \& Freytag, B. 2013, A\&A, 559, A104

Vanderbosch, Z., Hermes, J. J., Dennihy, E., et al. 2019, arXiv e-prints, arXiv:1908.09839

Vanderburg, A., Johnson, J. A., Rappaport, S., et al. 2015, Nature, 526, 546

van Sluijs, L., \& Van Eylen, V. 2018, MNRAS, 474, 4603

Williams, K. A., Bolte, M., \& Koester, D. 2009, ApJ, 693, 355

Zečević, P., Slater, C. T., Jurić, M., et al. 2019, AJ, 158, 37 